\section{OPEN ACCESS}

Edited by:

Carl G. Feng,

The University of Sydney, Australia

Reviewed by:

Amelia K. Pinto,

Saint Louis University, United States

Ali Zaid,

Griffith University, Australia

*Correspondence: Vivian Vasconcelos Costa vivianvcosta@gmail.com;

vivianvcosta@ufmg.br

Antonio Lucio Teixeira altexr@gmail.com

Specialty section: This article was submitted to Viral Immunology,

a section of the journal

Frontiers in Immunology

Received: 29 April 2021 Accepted: 30 June 2021

Published: 15 July 2021

Citation:

Marim FM, Teixeira DC, Queiroz-Junior CM, Valiate BVS,

Alves-Filho JC, Cunha TM,

Dantzer R, Teixeira MM, Teixeira AL and Costa W (2021) Inhibition

of Tryptophan Catabolism Is Associated With Neuroprotection

During Zika Virus Infection.

Front. Immunol. 12:702048. doi: 10.3389/fimmu.2021.702048

\title{
Inhibition of Tryptophan Catabolism Is Associated With Neuroprotection During Zika Virus Infection
}

\author{
Fernanda Martins Marim 1,2,3, Danielle Cunha Teixeira ${ }^{2,3}$, \\ Celso Martins Queiroz-Junior ${ }^{2,3,4}$, Bruno Vinicius Santos Valiate ${ }^{1,3}$, \\ Jose Carlos Alves-Filho ${ }^{5}$, Thiago Mattar Cunha ${ }^{5}$, Robert Dantzer ${ }^{6}$, \\ Mauro Martins Teixeira ${ }^{1,2,3}$, Antonio Lucio Teixeira $^{7 *}$ and Vivian Vasconcelos Costa ${ }^{2,3,4^{*}}$

\begin{abstract}
1 Department of Biochemistry and Immunology, Institute of Biological Sciences, Universidade Federal de Minas Gerais, Belo Horizonte, Brazil, 2 Research Group in Arboviral Diseases, Institute of Biological Sciences, Universidade Federal de Minas Gerais, Belo Horizonte, Brazil, ${ }^{3}$ Center for Drug Research and Development of Pharmaceuticals, Institute of Biological Sciences, Universidade Federal de Minas Gerais, Belo Horizonte, Brazil, 4 Departament of Morphology, Institute of Biological Sciences, Universidade Federal de Minas Gerais, Belo Horizonte, Brazil, ${ }^{5}$ Center for Research in Inflammatory Diseases (CRID), Department of Pharmacology, Ribeirao Preto Medical School, Universidade de São Paulo, Ribeirão Preto, Brazil, ${ }^{6}$ Department of Symptom Research, The University of Texas MD Anderson Cancer Center, Houston, TX, United States, 7 Department of Psychiatry and Behavioral Sciences, McGovern Medical Houston, The University of Texas Health Science Center at Houston, Houston, TX, United States
\end{abstract}

Zika virus (ZIKV) is an arbovirus belonging to Flaviviridae family that emerged as a global health threat due to its association with microcephaly and other severe neurological complications, including Guillain-Barré Syndrome (GBS) and Congenital Zika Syndrome (CZS). ZIKV disease has been linked to neuroinflammation and neuronal cell death. Neurodegenerative processes may be exacerbated by metabolites produced by the kynurenine pathway, an important pathway for the degradation of tryptophan, which induces neuronal dysfunction due to enhanced excitotoxicity. Here, we exploited the hypothesis that ZIKV-induced neurodegeneration can be rescued by blocking a target enzyme of the kynurenine pathway, the Indoleamine 2,3-dioxygenase (IDO-1). RT-PCR analysis showed increased levels of IDO-1 RNA expression in undifferentiated primary neurons isolated from wild type (WT) mice infected by ZIKV ex vivo, as well as in the brain of ZIKV-infected A129 mice. Pharmacological inhibition of IDO-1 enzyme with 1methyl-D-tryptophan (1-MT), in both in vitro and in vivo systems, led to significant reduction of ZIKV-induced neuronal death without interfering with the ability of ZIKV to replicate in those cells. Furthermore, in vivo analyses using both genetically modified mice $\left(\mathrm{IDO}^{-/-}\right.$mice) and A129 mice treated with 1-MT resulted in reduced microgliosis, astrogliosis and Caspase-3 positive cells in the brain of ZIKV-infected A129 mice. Interestingly, increased levels of CCL5 and CXCL-1 chemokines were found in the brain of 1-MT treated-mice. Together, our data indicate that IDO-1 blockade provides a 
neuroprotective effect against ZIKV-induced neurodegeneration, and this is amenable to inhibition by pharmacological treatment.

Keywords: Zika virus, IDO-1, neuroinflammation, neuronal death, microgliosis

\section{INTRODUCTION}

ZIKV is an arbovirus belonging to the family Flaviviridae, genus Flavivirus, composed of positive sense, single-stranded RNA. ZIKV was first isolated from a rhesus monkey in 1947 in the Zika forest (Uganda) and humans' infection was reported in Nigeria in 1954 (1). Epidemiological studies suggest that Zika virus had a wide geographical distribution, being introduced in Brazil between 2013 and 2015, causing a major epidemic (2) when there was an increase in cases of microcephaly and the incidence of Guillain-Barré syndrome (GBS) (3). ZIKV transmission occurs mainly by culicids of the genus Aedes (4), however, other transmission routes such as blood transfusion, sexual transmission and maternal-fetal transmission have already been demonstrated (5).

In humans, the disease caused by ZIKV manifests in about $20 \%$ of cases and is characterized by mild clinical symptoms such as fever, headache, myalgia, rash, conjunctivitis and joint pain (6). However, fetal brain samples from infected mothers have shown that ZIKV is able to break through biological placental barriers to infect developing neural cells inducing neuroinflammation, neuronal death, and neurodegeneration, therefore, leading to microcephaly and congenital Zika syndrome, both of which eventually manifest as fetal brain abnormalities (7-11).

The mechanism by which ZIKV exerts these neurological effects has been related to induction of neuronal excitotoxicity, a pathological process mediated by excessive glutamatergic activity (12), infection, activation, and apoptosis of neural progenitor cells, mature neurons, and glial cells with concomitant inflammation (13). Cell death and neurodegenerative processes can be exacerbated by metabolites produced by the kynurenine pathway (KP), an important pathway of degradation of tryptophan, at least in part due to excitotoxicity. Kynurenine pathway is induced by the activation of Indoleamine-2,3-dioxygenase (IDO-1) enzyme in the presence of proinflammatory cytokines, generating several neuroprotective and neurotoxic metabolites $(14,15)$.

IDO- 1 is found in macrophages, monocytes, microglia, astrocytes, and neurons $(16,17)$ and is considered the major enzyme involved in tryptophan degradation (18). Imbalances in levels of KP metabolites have been associated with neurodegenerative disorders including Huntington's, Alzheimer's, and Parkinson's diseases and other brain disorders as well as several cancers (19). IDO-1 has been linked to neurodegenerative diseases, and have been studied as a possible therapeutic target (19-23).

1-methyl-D-tryptophan (1-MT) is a methylated tryptophan, acting as a competitive IDO-1 inhibitor (24). Recent studies have shown its ability to improve responses to many anticancer therapies and to boost immunity in infectious diseases (24).
In this study we explored the hypothesis that ZIKV-induced neurodegeneration can be rescued by blocking the enzyme IDO1 that catalyzes the rate-limiting step in KP.

\section{MATERIAL AND METHODS}

\section{Animals and Ethics}

This study was carried out in accordance with the recommendations of the Brazilian Government (law 11794/ 2008a) and approved by the Committee on Animal Ethics of Universidade Federal de Minas Gerais (UFMG) (protocol no.106/2020 CEUA/UFMG). All mice were 5 to 8 weeks old and were kept under specific-pathogen-free conditions at $23^{\circ} \mathrm{C}$ on a 12-h light/12-h dark cycle with food and water provided $\mathrm{ad}$ libitum. Experiments were conducted using wild-type C57BL/6 mice, type I interferon receptor deficient mice (A129) on SV129/ Ev background, and deficient mice in the enzyme indoleamine 2,3-dioxygenase (IDO- $1^{-/-}$) on $\mathrm{C} 57 \mathrm{BL} / 6$ background at the Immunopharmacology Laboratory at ICB-UFMG. C57BL/6 mice were purchased from Bioterio Central of UFMG, A129 mice were originally purchased from B\&K Universal Limited (United Kingdom) and IDO- $1^{-/-}$were kindly provided by Universidade de São Paulo (USP).

\section{Mouse Experiments}

Mice were inoculated with ZIKV by intravenous (I.V) route (tail vein) with $4 \times 10^{3} \mathrm{PFU}$ of ZIKV in a volume of $200 \mu \mathrm{l}$ PBS or by intracranial (i.c) route with $1 \times 10^{6} \mathrm{PFU}$ of $\mathrm{ZIKV}$ in a volume of $20 \mu \mathrm{l}$ PBS. In some experiments, mice were treated orally with $10 \mathrm{mg}$ /animal of the Indoleamine 2,3-dioxygenase enzyme inhibitor (1-MT), 1 hour after infection and every 24 hours. Clinical symptoms were monitored daily and severely ill mice with a $\geq 20 \%$ weight decrease were euthanized. On day 5 after infection (the peak of ZIKV infection), the animals were euthanized to obtain spleen, brain, optic nerve and eye. For primary culture experiments (neurons), brains from mouse embryos on the fifteenth day of the embryonic period (E15) were collected to obtain neuronal cultures.

\section{Virus}

A low-passage-number clinical isolate of ZIKV (HS-2015-BA01 ), isolated from a viremic patient with symptomatic infection in Bahia State, Brazil, in 2015, was used. The complete genome of the virus is available at GenBank under the accession no. KX520666. Virus stocks were propagated in C6/36 Aedes albopictus cells and were titrated in CCL-81 Vero cells as described previously (25). 


\section{Intraocular Pressure Measurement (IOP)}

The IOP was measured on days 0,3 and 5 after ZIKV infection using an applanation tonometer (Tono-Pen Vet - Reichert Technologies, NY, USA), as previously described (26-28).

\section{Virus Titration}

The viral load on mouse tissues (brain, eye, optic nerve and spleen) and in the supernatant of cell culture samples was determined by plaque assay in CCL-81 Vero cells, as previously described (25). Plaque counts were computed as p.f.u. per gram of tissue mass or milliliter of supernatant.

\section{Evaluation of Inflammatory Markers}

The brain tissue was homogenized in a buffer containing protease inhibitors (100 mg of tissue per $1 \mathrm{~mL}$ of extraction solution; $0.4 \mathrm{~mol} / \mathrm{L}$ $\mathrm{NaCl}, 0.05 \%$ Tween $20,0.5 \%$ BSA, $0.1 \mathrm{mmol} / \mathrm{L}$ phenylmethyl sulfonil fluoride, $0.1 \mathrm{mmol} / \mathrm{L}$ benzethonium chloride, $10 \mathrm{mmol} / \mathrm{L}$ EDTA, and $20 \mathrm{KI}$ aprotinin). The brain homogenate was centrifuged at $3000 \times g$ for $10 \mathrm{~min}$ at $4^{\circ} \mathrm{C}$, and the supernatant was collected to determine the concentration of cytokines by ELISA. The cytokines (TNF and IL-1 $\beta$ ) and chemokines (CCL5 and CXCL1) levels in the brain of mice were measured using DuoSet ELISA kits antibodies (R\&D Systems) in accordance with the manufacturer's instructions. Neutrophil accumulation in mouse brains was also indirectly measured by determining the level of myeloperoxidase activity, according to a previous study (29).

\section{Brains Sections and Staining}

For histopathological scoring, brain samples were stained with hematoxylin and eosin ( $\mathrm{H} \& \mathrm{E})$ and the analyses was performed in cerebral cortex and hippocampus sections in a blinded manner according to previous studies $(29,30)$. Briefly, each brain region was graded on a four-point scale: 0 , no tissue damage; 1 , minimal tissue damage and/or mild inflammation; 2 , mild tissue damage and/or moderate inflammation; 3 , severe tissue damage and high inflammation; 4, necrosis with loss of tissue elements and presence of cellular debris. Meningeal inflammation was also graded on a four-point scale: 0, no inflammation; and points between 1 and 4 were assigned when there were one to four layers of cellular inflammation, respectively. The final score was calculated as a sum of cerebral cortex added to the score obtained from the meningeal inflammation analysis, totalizing a maximum of 8 points. For immunostaining analyses, brain samples were processed and sections from hippocampus, striatum, and prefrontal and motor cortex of mice were stained for microglia (IBA1 Polyclonal Antibody - Invitrogen), astrocytes (S100- $\beta$ polyclonal antibody - Abcam) and apoptosis (Caspase-3 Monoclonal Antibody - Invitrogen) according to manufacturer's instructions (Vector Elite kit Vector Laboratories) as described in as previously described (31). Image acquisition and analysis were performed using an Olympus BX 41 microscope (Olympus). The images presented in the article are representative of one of those experiments.

\section{Primary Neurons Cell Cultures}

Neuronal cultures were prepared from the cortex and striatal regions of E15 of C57BL/6 wild-type mouse embryo brains. After dissection, the brain tissue was submitted to trypsin digestion followed by cell dissociation using a fire-polished Pasteur pipette. Neuronal cells were plated onto poly-L-ornithine-coated dishes in neurobasal medium supplemented with N2 and B27 supplements (Gibco), $2 \mathrm{mM}$ GlutaMAX (Gibco) and $50 \mathrm{~g} / \mathrm{ml}$ penicillin/streptomycin (Sigma), incubated at $37^{\circ} \mathrm{C}$ and $5 \% \mathrm{CO}_{2}$ for 5 days. Infection was performed with ZIKV (MOI of 0.1), followed by an adsorption period of $1 \mathrm{~h}$. Wells were washed with incomplete medium and each well was replaced by a final volume of complete neurobasal medium. In some experiments, neuronal cultures were treated with $3,10,30$ or $100 \mu \mathrm{M}$ of the Indoleamine 2,3-dioxygenase enzyme inhibitor every $24 \mathrm{~h}$. Beside the kinetic experiments, all experiments evaluating the effects of 1-MT on primary neurons were performed after $48 \mathrm{~h}$ of ZIKV infection. At the time point, the supernatant was collected for quantification of viral load while the cells adhered to the plate were used in the cell death assay.

\section{Neuronal Cell Viability}

Neuronal cell death was assessed by LIVE/DEAD Cell Viability Assays after 48 hours of ZIKV infection, as previously described (27). Briefly, the neuronal cell culture was stained with $2 \mu \mathrm{M}$ calcein acetoxymethyl ester (AM) and $2 \mu \mathrm{M}$ ethidium homodimer -1. After 10 minutes, the neuronal culture was immediately visualized by a FLoid Cell Imaging Station fluorescence microscope (Thermo Fisher Scientific). Images were captured and the fractions of live (green, calcein AM) and dead (red, ethidium homodimer -1) cells in the same field of view were evaluated. Cell viability was analyzed, and the quantification was performed using the ImageJ software. Data are reported as the percentage of dead cells out of the total number of cells.

\section{Real-Time RT-PCR}

RNA was isolated using TRIzol ${ }^{\mathrm{TM}}$ reagent in accordance with the manufacturer's instructions (Invitrogen). RNA was resuspended in $30 \mu \mathrm{L}$ of nuclease-free water, and its concentration was analyzed by Nanodrop spectrophotometer. cDNAs were prepared from $2 \mu \mathrm{g}$ of total RNA extracted in a $10 \mu \mathrm{L}$ final reverse transcription reaction. RT-qPCR was performed from 10x diluted cDNA using SYBR Green PCR Master Mix (Applied Biosystems). Real-time was performed in a 7500 Fast Real-Time PCR System (Applied Biosystems). The appropriate primers were used to amplify a specific fragment corresponding to specific gene targets as follows: GAPDH F: 5'-ACG GCC GCA TCT TCT TGT GCA- 3'; GAPDH R: 5' -CGC CCA AAT CCG TTC ACA CCG A- 3; IDO-1 F: 5'-TCA AAG CAA TCC CCA CTG TAT CC- 3'; IDO-1 R: 5' -TCC ACA AAG TCA CGC ATC CTC- 3'. All data are presented as relative expression units after normalization to GAPDH gene, and measurements were conducted in duplicate.

\section{Statistical Analysis}

Results were expressed as Mean \pm SEM for the number of independent experiments. GraphPad Prism (GraphPad Software, Inc) was used to analyze data using different-tests as appropriate and $P<0.05, P<0.01, P<0.001$ and $P<0.0001$ 
indicate the levels of statistical significance. Additional information is indicated in Figure Legends.

\section{RESULTS}

\section{IDO-1 Expression Is Enhanced Upon ZIKV Infection In Vitro and In Vivo}

The KP is segregated into two distinct branches triggering neurotoxic and neuroprotective metabolites. The first step of $\mathrm{KP}$ converts the essential amino acid L-tryptophan to $\mathrm{N}$ formylkynurenine and is regulated by rate-limiting enzymes, being the Indoleamine 2,3-dioxygenase 1 (IDO-1) the main enzyme contributing to the production of kynurenines during inflammatory conditions (32). Therefore, differential mRNA expression of IDO-1 was investigated in non-infected and ZIKV-infected primary neuron cultures from cortex and striatal regions obtained from wild type (WT) C57BL/6 mice embryo brains. After 48 hours of ZIKV infection, there was an increase in the levels of IDO-1 when compared to Mock control in primary neuron cultures (Figure 1A). Additionally, increased levels of IDO-1 expression were also seen in the brain of A129 mice infected with ZIKV, 5 days upon infection, peak of disease manifestation in these mice, when compared to Mock littermates (Figure 1B). These results clearly demonstrate that ZIKV infection induced increased expression of IDO-1 enzyme in vitro and in vivo.

\section{1-MT Inhibitor Reduces Neuronal Death in ZIKV Primary Neurons}

Subsequently, we evaluated the effect of blockade of IDO-1 enzyme in vitro. Previous studies by our group have shown that undifferentiated neuronal cultures are highly susceptible to ZIKV infection, inducing neuronal death $(27,33)$. Then, we tested whether the IDO-I inhibitor, 1-methyl-D-tryptophan (1MT) would reduce ZIKV-induced neuronal cell death. For that, primary neuronal cultures obtained from the corticostriatal region of C57BL/6 embryo brains were infected with ZIKV (MOI 0.1 ) and treated with 1 -MT at concentrations of $3 ; 10$; 30 and $100 \mu \mathrm{M}$. After 48 hours following infection, the supernatant was collected to evaluate viral load by plaque assay and cells were subjected to cell viability assays. ZIKV neuronal infection resulted in $10^{9} \mathrm{PFU} / \mathrm{mL}$ in the culture supernatants, and in vitro IDO-1 inhibition with 1-MT did not interfere with ability of the virus to replicate in these cells. The highest 1-MT concentration triggered some reduction of the viral load (Figure 2A). Nevertheless, 1-MT treatment induced a significant reduction of neuronal cell death, in all evaluated concentrations (Figures 2B, C). At the concentration of 100 $\mu \mathrm{M}$ 1-MT, cytotoxicity was detected in infected cells, but without reaching statistical difference.

\section{IDO-1 Inhibition Did Not Prevent ZIKV-Induced Clinical Manifestations as Well as Virus Replication in A129 Mice}

Further, we investigated the effects of IDO-1 inhibition during ZIKV infection in vivo. For that, A129 mice were inoculated (i.v) with $4 \times 10^{3}$ PFU of ZIKV and, after 1 hour, they were treated with 1-MT and every 24 hours until euthanasia (Figure 3A). Five days after infection, peak of disease manifestation in this model, spleen, brain, optic nerve and eye of the animals were collected. In accordance with previous studies from our group (27), there was significant body weight loss in the ZIKV group when compared to Mock control. Similarly, infected mice treated
A

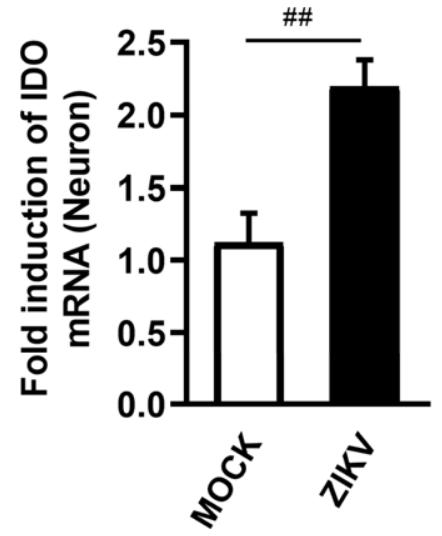

B

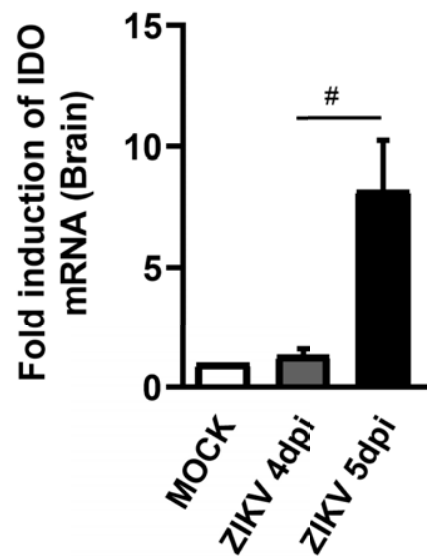

FIGURE 1 IDO-1 expression induced by ZIKV infection. (A) Primary culture of cortical-striatal neurons from C57BL/6 mice on D5 were infected with ZIKV (MOI 0.1) and after 48 hours the cells were harvested. (B) Type I interferon receptor deficient mice (A129) were inoculated (i.V) with $4 \times 10^{3}$ PFU/200 $\mu \mathrm{L}$ of ZIKV. At day 4 and 5 of infection, the brain was harvested. RNA extraction and subsequent Real time PCR analysis of IDO-1 expression in the brain and neuronal primary culture was done. Results are expressed as mean \pm SEM and are representative of two independent experiments. Statistically significant differences were assessed by Mann Whitney test. (\#) for $P<0.05$ and (\#\#) for $P<0.01$. 
A

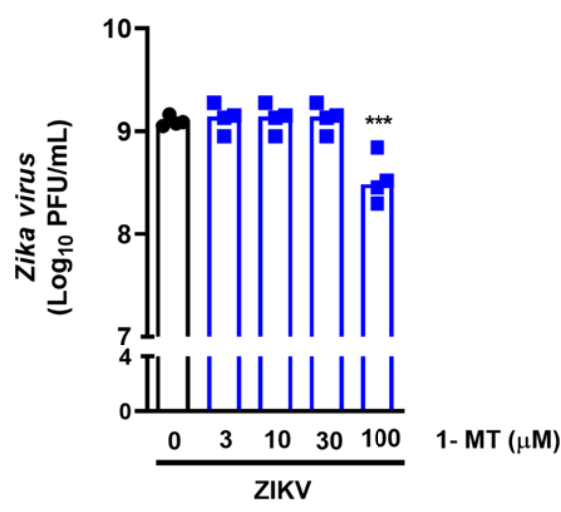

B

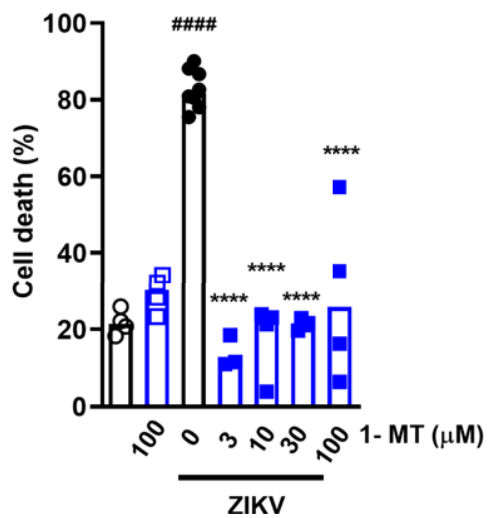

C

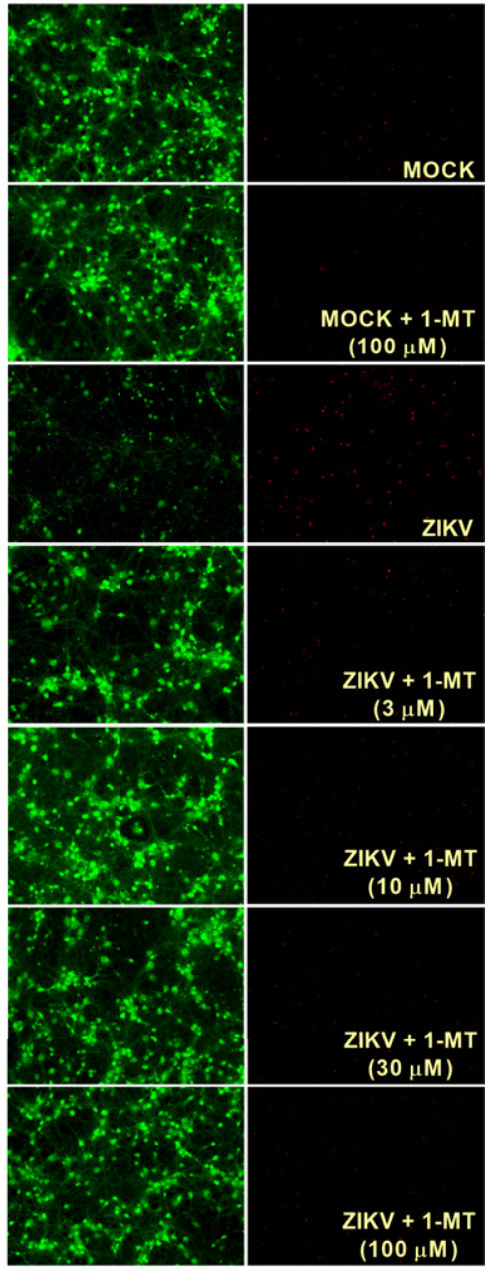

FIGURE 2 | IDO-1 inhibition in primary undifferentiated neurons infected by ZIKV. Primary culture of cortical-striatal neurons on D5. Neuronal culture from C57BL/6 mice were infected with ZIKV (MOI 0.1) and treated with 1-MT inhibitor at concentrations of 3; 10; 30 and $100 \mu \mathrm{M}$. After 48 hours, (A) the supernatant was collected to assess viral load, (B) cell death was assessed using the LIVE/DEAD Cell Viability Assay. (C) Representative images of infected and uninfected neurons stained with calcein AM (green indicates live cells) and ethidium homodimer (red indicates dead cells). All results are expressed as median and are representative of at least two independent experiments. Statistically significant differences were assessed by One-way ANOVA plus Holm-Sidak's or Tukey's comparisons test. (\#\#\#\#) for $P<0,0001$ compared to the MOCK group; $\left(^{(\star \star}\right)$ for $P<0,001$ and $\left(^{\star \star \star \star}\right)$ for $P<0,0001$ compared to the ZIKV group.

with 1-MT lost as much weight as the ZIKV group (Figure 3B). Ophthalmic alterations induced by ZIKV infection were also evaluated through the measurement of intraocular pressure (IOP), but no significant differences were detected between infected groups, treated or not with 1-MT (Figure 3C). Moreover, 1-MT treatment of ZIKV-infected mice did not interfere with viral loads detected in the brain (Figure 3D), optic nerve (Figure 3E), eye (Figure S1A) or spleen (Figure S1B). No significant differences were detected in neutrophils recruitment to the brain (Figure 3F). Although 1-MT did not prevent viral replication, it induced larger production of the chemokines CCL5 (Figure 3G) and CXCL1 (Figure 3H) in the brain of infected mice when compared to non-treated ZIKV group, but there was no difference in TNF (Figure 3I) and IL -1 $\beta$
(Figure 3J) levels. Overall, the results show that IDO-1 inhibition was not able to reduce clinical manifestations induced by ZIKVinfection, as well as virus replication in A129 mice.

\section{IDO-1 Inhibition Prevents Microgliosis, Astroglioses and Caspase-3 Expression of Cells in the Brain of ZIKV Infected Mice}

Infection of A129 mice with ZIKV triggered histopathological signs of brain inflammation and tissue damage. Histopathological analysis of the brain revealed mild gliosis and meningitis 5 days after ZIKV infection. Such changes were similar in non-treated and 1-MT-treated ZIKV-infected mice (Figures 4A, B). Despite such evidence, 1-MT treatment prompted a significant reduction in the number of IBA-1 
A

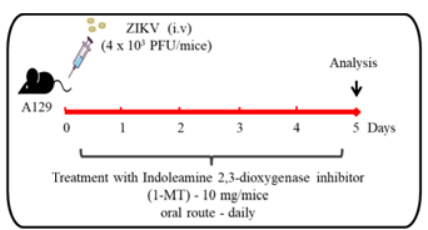

B

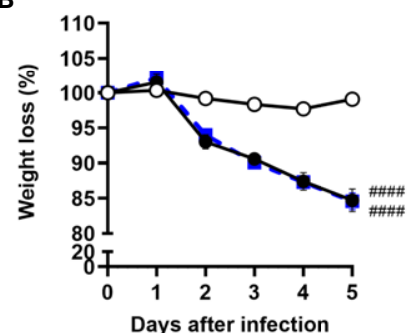

C

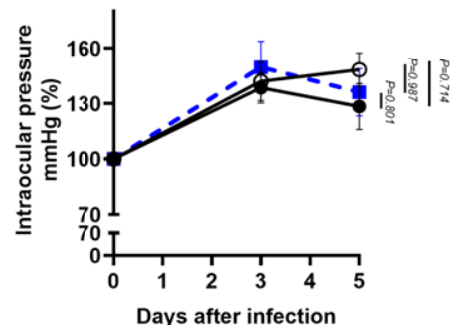

$\ominus$ MOCK $\rightarrow$ ZIKV

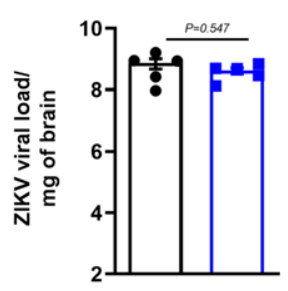

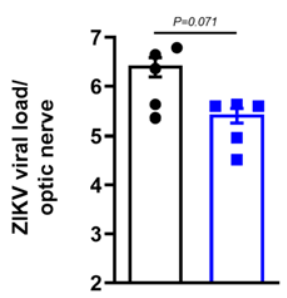

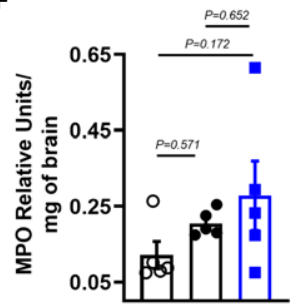

G

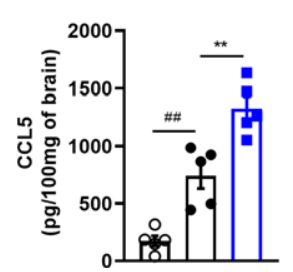

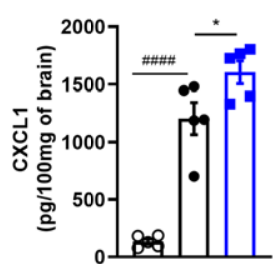

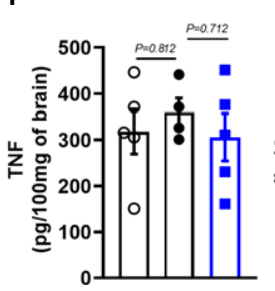

$\mathbf{J}$

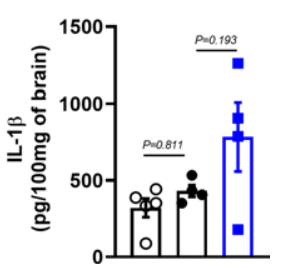

FIGURE 3 | Clinical signs and inflammatory parameters in A129 mice infected by ZIKV treated or not with 1-MT inhibitor. (A) Experimental schematic representation of experimental strategy. A129 were inoculated (i.v) with $4 \times 10^{3}$ PFU/200 $\mu \mathrm{L}$ of ZIKV and treated daily with 1-MT. (B) The body weight was evaluated daily and (C) the intraocular pressure was analyzed on days 0,3 and 5. At day 5 of infection, (D) the brain and (E) optical nerve was harvested for plaque assay analysis. (F) Measurement of myeloperoxidase activity (neutrophil accumulation) and (G-J) chemokine/cytokine production was assessed in mouse brain of animals. All results are expressed as mean and error bar indicate the standard error (SEM) and are representative of at least two independent experiments. Statistically significant differences were assessed by Two-way ANOVA plus Tukey's comparisons test (A, B), Mann Whitney test (D, E) and One-way ANOVA (F-J) plus Tukey's comparisons test. (\#\#) for $P<0,01$ and (\#\#\#\#) for $P<0,0001$ when compared to the MOCK group. (*) for $P<0,05$ and ${ }^{* \star}$ ) for $P<0,001$ compared to the ZIKV group.

positive cells (IBA- $1^{+}$cells) in the cerebral cortex of infected mice (Figures 4C, D). In addition, there was significant reduction in the number of astrocytes $\left(\mathrm{S} 100 \beta^{+}\right.$cells) in 1-MT-treated group when compared to non-treated ZIKV infected group (Figures 4E, F). Another important feature of ZIKV infection is neurodegeneration, including cell death. In this regard, treatment of infected mice with 1- MT decreased the number of brain Caspase-3-positive cells (Caspase- $3^{+}$cells), which is suggestive of reduced apoptosis (Figures 4G, H).

\section{Effects of ZIKV Infection in Indoleamine} 2,3-Dioxigenase $1\left(\right.$ IDO-1 $\left.1^{-/}\right)$Deficient Mice

In view of the effects observed after 1-MT treatment and the potential contribution of this pathway in preventing neurodegeneration, we also evaluated ZIKV infection in genetically modified Indoleamine 2,3-dioxigenase 1 deficient $\left(\mathrm{IDO}-1^{-1-}\right.$ ) mice. Since C57BL/6 mice are resistant to ZIKV infection $(27,34,35)$, ZIKV infection was performed by intracerebral route by inoculation of with $1 \times 10^{6} \mathrm{PFU}$ of 


\section{○ MOCK $\bullet$ ZIKV $\quad$ ZIKV + 1-MT}

A

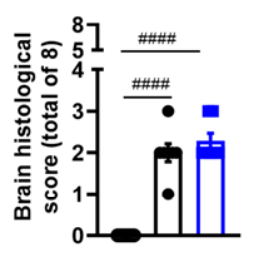

C

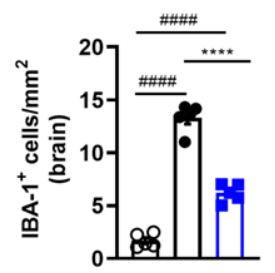

E

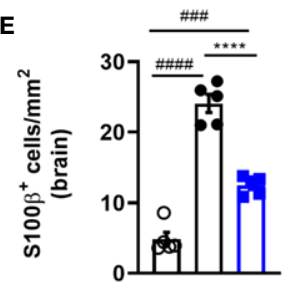

G

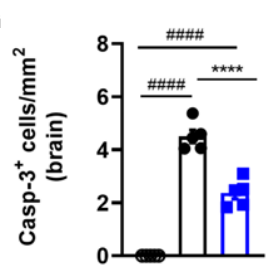

B

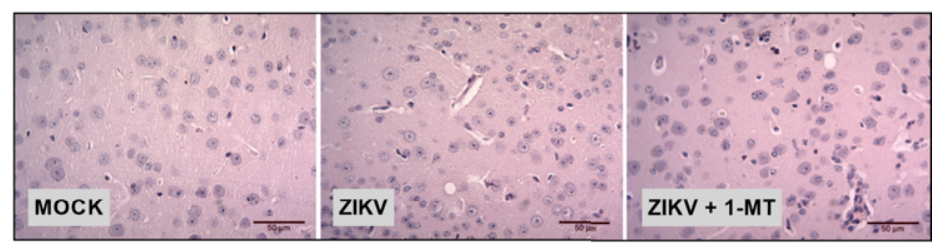

D

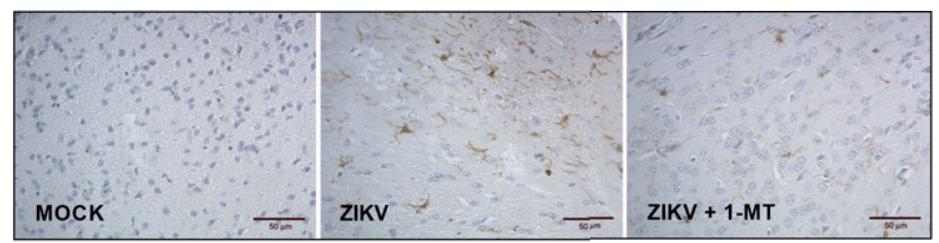

$\mathbf{F}$

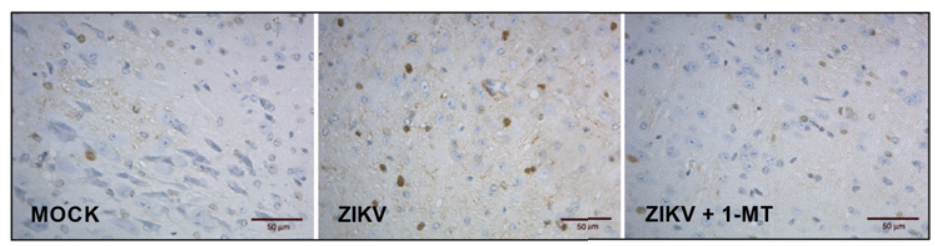

H

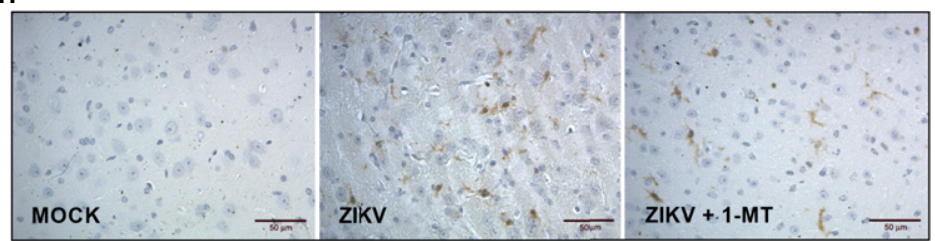

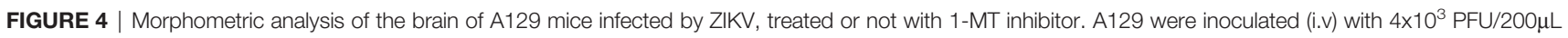
of ZIKV and treated daily with 1-MT. (A) Semiquantitative analysis (histopathological score) after H\&E staining of brain sections of ZIKV-infected mice 5 days after infection. (C, E, G) Immunostaining of (C) IBA- $1^{+}$, (E) S100- $\beta^{+}$and (G) Caspase-3 was performed in the brains of mice. (B, D, F, H) Representative images from brain sections. All results are expressed as mean and error bar indicate the standard error (SEM) and are representative of at least two independent experiments. Statistically significant differences were assessed by One-way ANOVA plus Tukey's comparisons test. (\#\#\#) for $P<0,001$ and (\#\#\#\#) for $P<0,0001$ when compared to the MOCK group. ${ }^{(\star \star \star)}$ for $P<0,0001$ compared to the ZIKV group.

ZIKV (i.c) in both WT C57BL/6 and IDO- $1^{-/-}$mice (Figure 5A). Body weight loss was significantly higher in C57BL/6 and IDO- ${ }^{-/-}$ mice infected with ZIKV than in their respective controls (Figure 5B). Furthermore, there was no significant change in the IOP levels between WT and IDO- $1^{-/-}$ZIKV-infected groups (Figure 5C). Regarding viral replication, no viable virus (assessed by plaque assay) or ZIKV genome (RT-PCR) was detected in the brain of ZIKV-infected WT or IDO- $1^{-/-}$mice. Accordingly, no difference in MPO levels was detected into mice's brains (Figure 5D). Finally, as demonstrated during 1-MT treatment, intracranial ZIKV infection also led to increased production of inflammatory mediators, such as CCL5 (Figure 5E) and CXCL1 (Figure 5F) in both WT and IDO-1 ${ }^{-/-}$ZIKV mice. Production of
TNF and IL-1 $\beta$ in WT and IDO- $1^{-/-}$was analogous to Mock (Figures 5G, H). Additionally, in a pattern similar to that observed in A129 mice treated with 1-MT, brain histopathological analysis of C57BL/6 and IDO- $1^{-/-}$infected mice revealed mild gliosis and meningitis in both infected experimental groups (Figures 6A, B). Accordingly, reduced microgliosis, astrogliosis and apoptosis were found in infected IDO- $1^{-/-}$mice in comparison to ZIKV infected WT littermates as demonstrated by reduction of the number of IBA-1 (Figures 6C, D), S100 $\beta$ (Figures 6E, F) and Caspase-3 (Figures 6G, H) positive cells of mice's brain sections. Furthermore, the morphology of the Caspase-3-stained cells, especially the nucleus morphology, comprised both neurons and glial cells. 
A

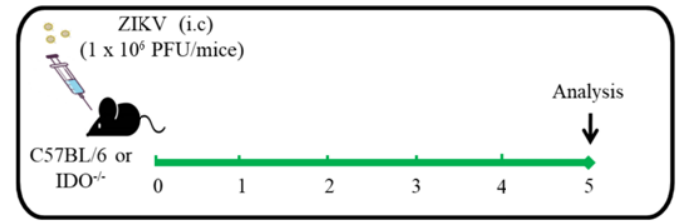

B

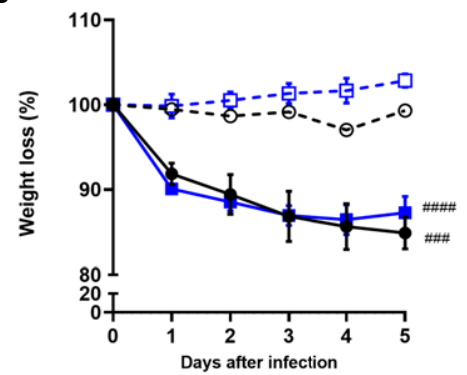

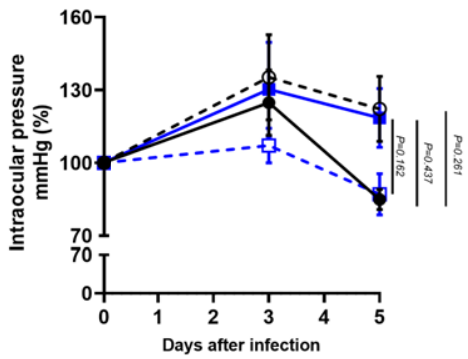
C57BL/6 MOCK
C57BL/6 ZIKV
七 $\mathrm{IDO}^{-/-} \mathrm{MOCK}$
$\mathrm{IDO}^{-/ 2} \mathrm{ZIKV}$

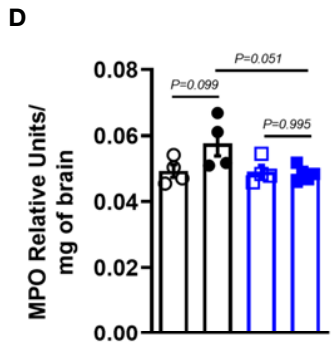

E

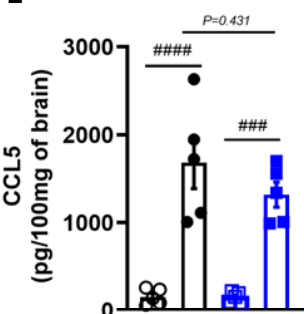

F

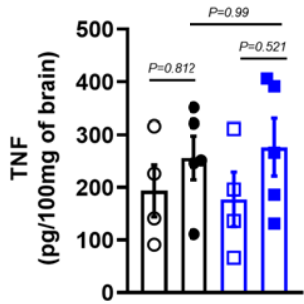

H

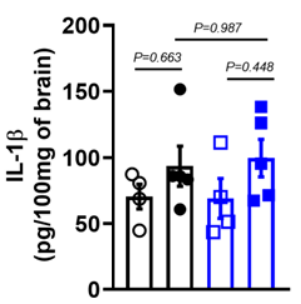

FIGURE 5 | Clinical signs and inflammatory parameters after ZIKV infection of WT and IDO-/- mice infected by ZIKV. (A) Experimental schematic representation of experimental strategy. C57BL/6 and IDO $-1^{-/-}$were inoculated (intracranial) with $1 \times 10^{6} \mathrm{PFU} / 20 \mu \mathrm{L}$ of ZIKV. (B) The body weight was evaluated daily and (C) the intraocular pressure was analyzed on days 0,3 and 5 . At day 5 of infection, (D) the brain was harvested and myeloperoxidase absorbance analysis and (E-H) chemokine/cytokine production was assessed. All results are expressed as mean and error bar indicate the standard error (SEM) and are representative of at least two independent experiments. Statistically significant differences were assessed by Two-way ANOVA plus Tukey's comparisons test (B, C) and One-way ANOVA plus Tukey's comparisons test (D-H). (\#\#\#) for $P<0,001$ and (\#\#\#\#) for $P<0,0001$ compared to the MOCK group.

\section{DISCUSSION}

Several studies have reported the tropism of the ZIKV to different cellular types, tissues and body fluids. Meanwhile, ZIKV-tropism to the CNS is the most harmful feature to the host and may cause neurodegeneration and other neuronal dysfunctions. Despite its association with neurological diseases, such as microcephaly, GBS and CZS, there are no current approved therapies for the treatment of this important pathogen (36). In order to develop effective therapies, a better understanding of the pathogenicity resulting from the virus-host interaction is required. Global analysis of gene expression shows that ZIKV infection prompts significant dysregulation of several host factors associated with neural development, immune response, cell death, among others $(37,38)$. In the present study, we evaluated the potential neuroprotective effect of the blockade of IDO-1 enzyme by using a genetically approach (IDO- $1^{-/-}$mice) or a pharmacological strategy (1-MT inhibitor), in vitro and in vivo. The main results 

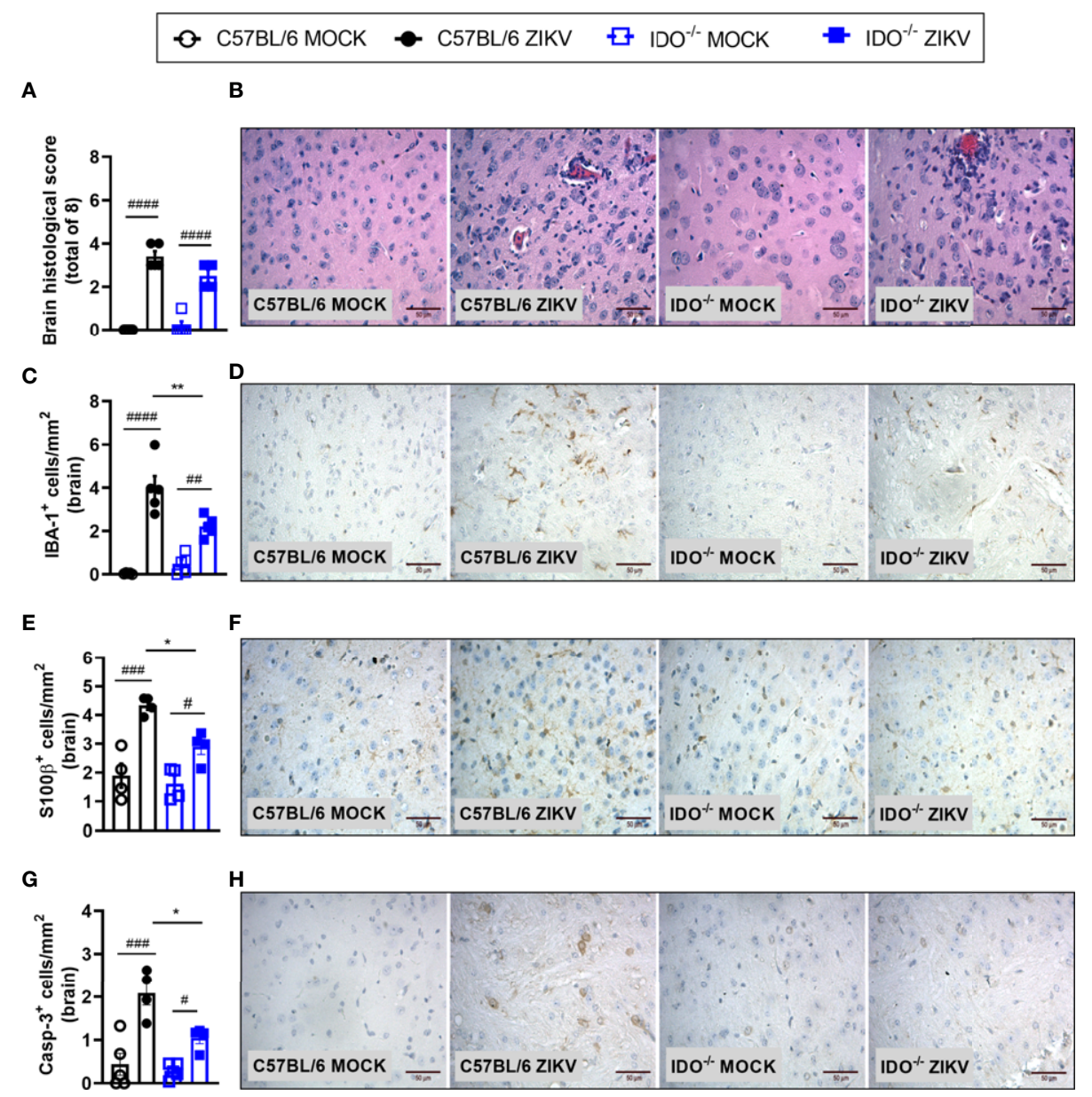

FIGURE 6 | Morphometric analysis of the brain of WT and IDO-/ mice infected by ZIKV. C57BL/6 and IDO-1/- were inoculated (intracranial) with $1 \times 10^{6} \mathrm{PFU} / 20 \mu \mathrm{L}$ of ZIKV. (A) Semiquantitative analysis (histopathological score) after H\&E staining of brain sections of ZIKV-infected mice 5 days after infection. (C, E, G)

Immunostaining of (C) IBA- $1^{+}$, (E) S100- $\beta^{+}$and (G) Caspase-3 was performed in the brains of mice. (B, D, F, H) Representative images from brain sections. Results are expressed as mean and error bar indicate the standard error (SEM) and are representative of at least two independent experiments. Statistically significant differences were assessed by One-way ANOVA plus Tukey's comparisons test (\#) for $P<0,05$, (\#\#) for $P<0,01$ and (\#\#\#) for $P<0,001$ and (\#\#\#\#) for $P<0,0001$ when compared to the MOCK group. (*) for $P<0,05$ and $\left(^{(\star)}\right)$ for $P<0,01$ compared to the ZIKV group.

were: (I) increased levels of IDO-1 expression after ZIKV infection; (II) reduction of neuronal death upon 1-MT treatment; (III); massive reduction of ZIKV-induced microgliosis, astrogliosis and apoptosis in $\mathrm{IDO}^{-/-}$mice and after 1-MT administration in A129 mice. Overall, these data indicate a neuroprotective effect induced by IDO-1 inhibition after ZIKV infection in vivo and in vitro.

IDO-1 is the first rate-limiting of the KP responsible for converting tryptophan into several downstream kynurenine metabolites. This enzyme is expressed by many cell types, including macrophages, microglia, dendritic cells, astrocytes, fibroblasts, and epithelial cells (32) and is commonly induced by proinflammatory cytokines. In the current study, we showed that ZIKV infection increased levels of IDO-1 expression in mice's brain and also in primary neuron culture obtained from embryonic brains of mice. These results demonstrate that Indoleamine-2,3-dioxygenase, or IDO-1, is activated by the inflammatory response induced by ZIKV. In this context, we next, evaluated the effects of the inhibition of IDO-1 on ZIKV infection in vitro and in vivo systems. We found that a pharmacological inhibition in primary neurons infected by ZIKV did not prevent viral replication but induced less cell death. Likewise, human monocyte-derived macrophages induce IDO-1 expression upon West Nile virus (WNV), via TNF and NF-KB signaling, but IDO was not essential for the control of WNV infection (39). Similarly, 1-MT treatment of ZIKVinfected mice did not interfere with the ability of ZIKV to replicate in the brain, optic nerve, eye or spleen tissues. Additionally, no significant difference in neutrophil recruitment was observed between treated and non-treated ZIKV-infected mice. At the same time, chemokines relevant for the migration of monocytes, lymphocytes, as well as for neutrophil traffic, such as CCL5 and CXCL1, respectively, had 
increased levels in 1-MT treated ZIKV mice. We also evaluated ZIKV infection in IDO- $1^{-/-}$mice and WT mouse model. Corroborating the pharmacological data, the absence of IDO did not interfere with clinical signs, viral replication or inflammatory parameters, although microgliosis, astrogliosis and caspase- 3 expression reduced after infection. Chemokine signaling plays an important homeostatic role and modulates neuroprotective processes on different stimuli, orchestrating the action of neurons-microglia-astrocytes to preserve brain damage (40). In pathological processes, pro-inflammatory cytokines and chemokines are robustly induced and can lead IDO-1 activity and tryptophan associated metabolites (39, 41, 42). IDO-1 inhibition in Influenza virus infection does not affect leukocyte infiltration or viral clearance, thus, blockade of IDO-1 could play a beneficial role in host protection (43-45). These data show that pharmacological inhibition or genetic ablation of IDO-1 did not completely prevent brain inflammation, but reduced brain damage and cell death.

ZIKV can infect almost all CNS cells, including neurons, glial cells, endothelial cells and pericytes. Activated CNS-resident cells could be the source of proinflammatory cytokines and chemokines such as CCL5 and CXCL1, found in ZIKVinfected brain tissue. ZIKV infection and replication are associated with brain damage and, as consequence, severe neurological symptoms $(8,27)$. The mechanisms by which ZIKV exert these effects involve neuronal excitotoxicity and apoptosis of microglia, astrocytes and other cells. Our group has shown that neuronal cell death induced by ZIKV is associated with excitotoxicity mediated by the release of glutamate and other neurotoxic factors such as TNF and IL$1 \beta$, inducing the activation of NMDA (N-methyl-d-aspartate receptor) receptors $(27,33)$. Accordingly, treatment with Memantine (an FDA-approved allosteric NMDA receptor antagonist) in vivo and in vitro was effective to prevent ZIKVinduced cell death and neurodegeneration but did not interfere with the ability of the virus to replicate. Furthermore, administration of Ifenprodil (a selective inhibitor of the NMDA receptor, specifically for the GluN1 and GluN2B subunits) in a primary neuron culture rescues ZIKV-induced neurotoxicity $(27,33)$. IDO expression in Huntington's disease is chronically elevated, inducing neurotoxicity, and IDO inhibition reduces neurotoxicity sensitivity and neuroprotective role (46).

Cell death and neurodegenerative processes can be exacerbated by metabolites produced by KP. Kynurenine metabolism is directly associated with several neurodegenerative and neurological diseases, such as Alzheimer's disease, multiple sclerosis, and stroke $(23,47)$. In accordance with recent studies from our group $(27,33,48)$, infection of A129 mice induced brain inflammation and tissue damage, and we found mild gliosis and meningitis in all infected mice groups. However, pharmacological inhibition or deletion of IDO-1 (IDO ${ }^{-/}$) prompted a significant reduction of IBA-1 positive cells in the brain's mice, suggesting less microglia activation. It appears that microglia activation is dependent on IDO-1 $(49,50)$. In a similar pattern, we found a reduction in the number of astrocytes ( $\mathrm{S} 100 \beta$ positive cells). Microglia and activated astrocytes produce several inflammatory mediators, including IL-1 $\beta$, TNF, glutamate, nitric oxide (NO) among others, which can trigger neuronal death after ZIKV infection (33). In addition, ZIKV-induced neurodegeneration can be caused by loss of structure or function of neurons. Recent studies demonstrated that ZIKV is able to induce apoptosis of neuronal cells in vitro (33) and in vivo (28). In this regard, we have shown that treatment of infected mice with 1-MT decreased the number of brain Caspase-3positive cells, which is suggestive of reduced apoptosis. According to cell morphology, especially the nucleus, these cells comprised both neurons and glial cells, however, co-localization assays were not performed (Caspase-3/IBA-1 or Caspase-3/S100 $\beta$ or NeuN/Caspase-3). Although ZIKV-induces neuronal damage, it is unclear whether neuronal death is due to direct neurotoxicity or secondary to the activation of microglia and astrocytes. Apoptosis can be a result from inflammatory mediators released by the activated microglia or induced in a non-cell autonomous manner, triggering cell death of uninfected neurons by releasing cytotoxic factors (33). These data suggest the pharmacological or genetic inhibition of IDO-1 prevents microglial activation, astrogliosis and apoptosis in the brain of ZIKV infected, without preventing clinical outcomes.

Development of efficient therapies against viral infections, such as ZIKV, is important for the control and reduction of clinical symptoms, viral load, neuroinflammation, and mainly, neurodegeneration. A combination of compounds that could prevent ZIKV-induced neurodegeneration with viral replication (antiviral drugs) represents the ideal treatment. Meanwhile, no approved antiviral drugs are available. Recently, our group showed that a therapeutic administration of an antiviral peptide (AH-D) significantly reduced viral loads in serum, spleen, brain and optical nerve throughout the course of ZIKV infection (30). Additionally, we also demonstrated the efficacy of the 7-Deaza-7-fluoro-2'-C-methyl adenosine (DFMA), a nucleoside analogue, which showed significant antiviral effects against ZIKV in vitro and in vivo systems, especially when administered prophylactically and at early times after ZIKV infection (48). These compounds open a new perspective to evaluate the synergic effect of a neuroprotective drug such as 1MT along with an antiviral drug against ZIKV-induced disease in an early future.

In conclusion, our data indicate that IDO- 1 inhibition exerts a neuroprotective role in CNS reducing the microglial activation, astrogliosis and apoptosis without antiviral effect. Further studies are required to elucidate the mechanisms of neuroprotection obtained by ablation of IDO-1 and it will be important to evaluate whether the combination of neuroprotective drugs, such 1-MT, and antiviral like AH-D or DFMA could be the treatment for ZIKV infection.

\section{DATA AVAILABILITY STATEMENT}

The raw data supporting the conclusions of this article will be made available by the authors, without undue reservation. 


\section{ETHICS STATEMENT}

The animal study was reviewed and approved by Committee on Animal Ethics of Universidade Federal de Minas Gerais (UFMG) (protocol no.106/2020 CEUA/UFMG).

\section{AUTHOR CONTRIBUTIONS}

FM, JA-F, TC, MT, AT, and VC designed the study. FM, DT, CQ-J, and BV conducted the experiments and analysis of the data. FM, AT, and VC interpreted the results. FM, CQ-J, AT, and VC wrote the first draft of the paper. RD, MT, AT, and VC obtained funding. All authors contributed to the article and approved the submitted version.

\section{FUNDING}

This work was supported by the Grants from National Institute of Science and Technology in Dengue and Host-microorganism Interaction (INCT dengue), which is a programme sponsored by the Brazilian National Science Council (CNPq, Brazil) and the Minas Gerais Foundation for Science (FAPEMIG, Brazil). This work also received support from Coordenação de Aperfeiçoamento de Pessoal de Nivel Superior - CAPES/Brazil (grant no CAPES: 88881.130741), ZIKALLIANCE consortium 734548, Financiadora de Estudos e Pesquisa (FINEP

\section{REFERENCES}

1. Macnamara FN. Zika Virus: A Report on Three Cases of Human Infection During an Epidemic of Jaundice in Nigeria. Trans R Soc Trop Med Hyg (1954) 48(2):139-45. doi: 10.1016/0035-9203(54)90006-1

2. Baud D, Gubler DJ, Schaub B, Lanteri MC, Musso D. An Update on Zika Virus Infection. Lancet (2017) 390(10107):2099-109. doi: 10.1016/S01406736(17)31450-2

3. Campos GS, Bandeira AC, Sardi SI. Zika Virus Outbreak, Bahia, Brazil. Emerg Infect Dis (2015) 21(10):1885-6. doi: 10.3201/eid2110.150847

4. Cunha MS, Esposito DL, Rocco IM, Maeda AY, Vasami FG, Nogueira JS, et al. First Complete Genome Sequence of Zika Virus (Flaviviridae, Flavivirus) From an Autochthonous Transmission in Brazil. Genome Announc (2016) 4 (2):e00032-16. doi: 10.1128/genomeA.00032-16

5. Kurscheidt FA, Mesquita CSS, Damke G, Damke E, Carvalho A, Suehiro TT, et al. Persistence and Clinical Relevance of Zika Virus in the Male Genital Tract. Nat Rev Urol (2019) 16(4):211-30. doi: 10.1038/s41585-019-0149-7

6. Duffy MR, Chen TH, Hancock WT, Powers AM, Kool JL, Lanciotti RS, et al. Zika Virus Outbreak on Yap Island, Federated States of Micronesia. N Engl J Med (2009) 360(24):2536-43. doi: 10.1056/NEJMoa0805715

7. Cugola FR, Fernandes IR, Russo FB, Freitas BC, Dias JL, Guimaraes KP, et al. The Brazilian Zika Virus Strain Causes Birth Defects in Experimental Models. Nature (2016) 534(7606):267-71. doi: 10.1038/nature18296

8. Garcez PP, Loiola EC, Madeiro da Costa R, Higa LM, Trindade P, Delvecchio R, et al. Zika Virus Impairs Growth in Human Neurospheres and Brain Organoids. Science (2016) 352(6287):816-8. doi: 10.1126/ science.aaf6116

9. Noronha L, Zanluca C, Azevedo ML, Luz KG, Santos CN. Zika Virus Damages the Human Placental Barrier and Presents Marked Fetal Neurotropism. Mem Inst Oswaldo Cruz (2016) 111(5):287-93. doi: 10.1590/0074-02760160085

10. Dallmeier K, Neyts J. Zika and Other Emerging Viruses: Aiming at the Right Target. Cell Host Microbe (2016) 20(4):420-2. doi: 10.1016/j.chom.2016.09.011
01.16.0050.00, Brazil), Conselho Nacional de Desenvolvimento Cientifico e Tecnológico (CNPq) under grants: 425359/2018 and 440423/2016-13, Fundação de Amparo à Pesquisa do Estado de Minas Gerais (FAPEMIG, Brazil) - APQ-02281- 18) and by L'Oréal-Unesco-ABC For Women in Science Program). Also, this work was funded by NIH (R01 CA193522 and R01 NS073939) and an MD Anderson Cancer Support Grant (P30 CA016672).

\section{ACKNOWLEDGMENTS}

We thank Ilma Marçal and Tania Colina for technical support during the study.

\section{SUPPLEMENTARY MATERIAL}

The Supplementary Material for this article can be found online at: https://www.frontiersin.org/articles/10.3389/fimmu.2021. 702048/full\#supplementary-material

Supplementary Figure 1 | Viral load in the eye and spleen of A129 mice infected by ZIKV, treated or not with 1-MT inhibitor. A129 were inoculated (i.v) with $4 \times 10^{3}$ $\mathrm{PFU} / 200 \mu \mathrm{L}$ of ZIKV and treated daily with 1-MT. (A) Eye and (B) spleen was harvested for plaque assay analysis. All results are expressed as mean and error bar indicate the standard error (SEM) and are representative of at least two independent experiments. Statistically significant differences were assessed by One-way ANOVA plus Tukey's comparisons test.

11. Saiz JC, Martin-Acebes MA. The Race To Find Antivirals for Zika Virus Antimicrob Agents Chemother (2017) 61(6):e00411-17. doi: 10.1128/AAC.00411-17

12. Dong XX, Wang Y, Qin ZH. Molecular Mechanisms of Excitotoxicity and Their Relevance to Pathogenesis of Neurodegenerative Diseases. Acta Pharmacol Sin (2009) 30(4):379-87. doi: 10.1038/aps.2009.24

13. Marim FM, Camargos VN, Queiroz-Junior CM, Costa VV. Zika Virus Infection and Potential Mechanisms Implicated in Neuropsychiatric Complications. In: AL Teixeira, D Macedo, BT Baune, editors. Perinatal Inflammation and Adult Psychopathology: From Preclinical Models to Humans. Springer (2020). p. 207-22.

14. O'Connor JC, Andre C, Wang Y, Lawson MA, Szegedi SS, Lestage J, et al. Interferon-Gamma and Tumor Necrosis Factor-Alpha Mediate the Upregulation of Indoleamine 2,3-Dioxygenase and the Induction of Depressive-Like Behavior in Mice in Response to Bacillus Calmette-Guerin. J Neurosci (2009) 29(13):4200-9. doi: 10.1523/JNEUROSCI.5032-08.2009

15. Campbell BM, Charych E, Lee AW, Moller T. Kynurenines in CNS Disease: Regulation by Inflammatory Cytokines. Front Neurosci (2014) 8:12. doi: 10.3389/fnins.2014.00012

16. Guillemin GJ, Cullen KM, Lim CK, Smythe GA, Garner B, Kapoor V, et al. Characterization of the Kynurenine Pathway in Human Neurons. J Neurosci (2007) 27(47):12884-92. doi: 10.1523/JNEUROSCI.4101-07.2007

17. Jones SP, Guillemin GJ, Brew BJ. The Kynurenine Pathway in Stem Cell Biology. Int J Tryptophan Res (2013) 6:57-66. doi: 10.4137/IJTR.S12626

18. Lob S, Konigsrainer A, Zieker D, Brucher BL, Rammensee HG, Opelz G, et al. IDO1 and IDO2 Are Expressed in Human Tumors: Levo- But Not Dextro-1Methyl Tryptophan Inhibits Tryptophan Catabolism. Cancer Immunol Immunother (2009) 58(1):153-7. doi: 10.1007/s00262-008-0513-6

19. Maddison DC, Giorgini F. The Kynurenine Pathway and Neurodegenerative Disease. Semin Cell Dev Biol (2015) 40:134-41. doi: 10.1016/j.semcdb.2015.03.002

20. Zadori D, Klivenyi P, Szalardy L, Fulop F, Toldi J, Vecsei L. Mitochondrial Disturbances, Excitotoxicity, Neuroinflammation and Kynurenines: Novel Therapeutic Strategies for Neurodegenerative Disorders. J Neurol Sci (2012) 322(1-2):187-91. doi: 10.1016/j.jns.2012.06.004 
21. Giil LM, Midttun O, Refsum H, Ulvik A, Advani R, Smith AD, et al. Kynurenine Pathway Metabolites in Alzheimer's Disease. J Alzheimers Dis (2017) 60(2):495-504. doi: 10.3233/JAD-170485

22. Hestad KA, Engedal K, Whist JE, Farup PG. The Relationships Among Tryptophan, Kynurenine, Indoleamine 2,3-Dioxygenase, Depression, and Neuropsychological Performance. Front Psychol (2017) 8:1561. doi: 10.3389/ fpsyg.2017.01561

23. Colpo GD, Venna VR, McCullough LD, Teixeira AL. Systematic Review on the Involvement of the Kynurenine Pathway in Stroke: Pre-Clinical and Clinical Evidence. Front Neurol (2019) 10:778. doi: 10.3389/fneur.2019.00778

24. Fox E, Oliver T, Rowe M, Thomas S, Zakharia Y, Gilman PB, et al. Indoximod: An Immunometabolic Adjuvant That Empowers T Cell Activity in Cancer. Front Oncol (2018) 8:370. doi: 10.3389/fonc.2018.00370

25. Costa VV, Fagundes CT, Valadao DF, Avila TV, Cisalpino D, Rocha RF, et al. Subversion of Early Innate Antiviral Responses During Antibody-Dependent Enhancement of Dengue Virus Infection Induces Severe Disease in Immunocompetent Mice. Med Microbiol Immunol (2014) 203(4):231-50. doi: 10.1007/s00430-014-0334-5

26. Foureaux G, Franca JR, Nogueira JC, Fulgencio Gde O, Ribeiro TG, Castilho RO, et al. Ocular Inserts for Sustained Release of the Angiotensin-Converting Enzyme 2 Activator, Diminazene Aceturate, to Treat Glaucoma in Rats. PloS One (2015) 10(7):e0133149. doi: 10.1371/journal.pone.0133149

27. Costa VV, Del Sarto JL, Rocha RF, Silva FR, Doria JG, Olmo IG, et al. N-Methyl-DAspartate (NMDA) Receptor Blockade Prevents Neuronal Death Induced by Zika Virus Infection. mBio (2017) 8(2):e00350-17. doi: 10.1128/mBio.00350-17

28. Camargos VN, Foureaux G, Medeiros DC, da Silveira VT, Queiroz-Junior CM, Matosinhos ALB, et al. In-Depth Characterization of Congenital Zika Syndrome in Immunocompetent Mice: Antibody-Dependent Enhancement and an Antiviral Peptide Therapy. EBioMedicine (2019) 44:516-29. doi: 10.1016/j.ebiom.2019.05.014

29. Amaral DC, Rachid MA, Vilela MC, Campos RD, Ferreira GP, Rodrigues DH, et al. Intracerebral Infection With Dengue-3 Virus Induces Meningoencephalitis and Behavioral Changes That Precede Lethality in Mice. J Neuroinflamm (2011) 8:23. doi: 10.1186/1742-2094-8-23

30. Jackman JA, Costa VV, Park S, Real A, Park JH, Cardozo PL, et al. Therapeutic Treatment of Zika Virus Infection Using a Brain-Penetrating Antiviral Peptide. Nat Mater (2018) 17(11):971-7. doi: 10.1038/s41563-018-0194-2

31. Doria JG, de Souza JM, Silva FR, Olmo IG, Carvalho TG, Alves-Silva J, et al. The mGluR5 Positive Allosteric Modulator VU0409551 Improves Synaptic Plasticity and Memory of a Mouse Model of Huntington's Disease. J Neurochem (2018) 147(2):222-39. doi: 10.1111/jnc.14555

32. Fujigaki H, Yamamoto Y, Saito K. L-Tryptophan-Kynurenine Pathway Enzymes are Therapeutic Target for Neuropsychiatric Diseases: Focus on Cell Type Differences. Neuropharmacology (2017) 112(Pt B):264-74. doi: 10.1016/j.neuropharm.2016.01.011

33. Olmo IG, Carvalho TG, Costa VV, Alves-Silva J, Ferrari CZ, Izidoro-Toledo TC, et al. Zika Virus Promotes Neuronal Cell Death in a Non-Cell Autonomous Manner by Triggering the Release of Neurotoxic Factors. Front Immunol (2017) 8:1016. doi: 10.3389/fimmu.2017.01016

34. Rossi SL, Tesh RB, Azar SR, Muruato AE, Hanley KA, Auguste AJ, et al. Characterization of a Novel Murine Model to Study Zika Virus. Am J Trop Med Hyg (2016) 94(6):1362-9. doi: 10.4269/ajtmh.16-0111

35. Dowall SD, Graham VA, Rayner E, Atkinson B, Hall G, Watson RJ, et al. A Susceptible Mouse Model for Zika Virus Infection. PloS Negl Trop Dis (2016) 10(5):e0004658. doi: 10.1371/journal.pntd.0004658

36. Boldescu V, Behnam MAM, Vasilakis N, Klein CD. Broad-Spectrum Agents for Flaviviral Infections: Dengue, Zika and Beyond. Nat Rev Drug Discov (2017) 16(8):565-86. doi: 10.1038/nrd.2017.33

37. Sun $X$, Hua S, Chen HR, Ouyang Z, Einkauf K, Tse S, et al. Transcriptional Changes During Naturally Acquired Zika Virus Infection Render Dendritic Cells Highly Conducive to Viral Replication. Cell Rep (2017) 21(12):3471-82. doi: 10.1016/j.celrep.2017.11.087
38. Chang Y, Jiang Y, Li C, Wang Q, Zhang F, Qin CF, et al. Different Gene Networks are Disturbed by Zika Virus Infection in a Mouse Microcephaly Model. Genomics Proteomics Bioinf (2021). doi: 10.1016/j.gpb.2019.06.004

39. Yeung AW, Wu W, Freewan M, Stocker R, King NJ, Thomas SR. Flavivirus Infection Induces Indoleamine 2,3-Dioxygenase in Human MonocyteDerived Macrophages Via Tumor Necrosis Factor and NF-kappaB. J Leukoc Biol (2012) 91(4):657-66. doi: 10.1189/jlb.1011532

40. Trettel F, Di Castro MA, Limatola C. Chemokines: Key Molecules That Orchestrate Communication Among Neurons, Microglia and Astrocytes to Preserve Brain Function. Neuroscience (2020) 439:230-40. doi: 10.1016/ j.neuroscience.2019.07.035

41. Ito H, Ando T, Ogiso H, Arioka Y, Saito K, Seishima M. Inhibition of Indoleamine 2,3-Dioxygenase Activity Accelerates Skin Wound Healing. Biomaterials (2015) 53:221-8. doi: 10.1016/j.biomaterials.2015.02.098

42. Hoshi M, Osawa Y, Ito H, Ohtaki H, Ando T, Takamatsu M, et al. Blockade of Indoleamine 2,3-Dioxygenase Reduces Mortality From Peritonitis and Sepsis in Mice by Regulating Functions of CD11b+ Peritoneal Cells. Infect Immun (2014) 82(11):4487-95. doi: 10.1128/IAI.02113-14

43. Fox JM, Sage LK, Huang L, Barber J, Klonowski KD, Mellor AL, et al. Inhibition of Indoleamine 2,3-Dioxygenase Enhances the T-Cell Response to Influenza Virus Infection. J Gen Virol (2013) 94(Pt 7):1451-61. doi: 10.1099/vir.0.053124-0

44. Lin YT, Lin CF, Yeh TH. Influenza A Virus Infection Induces Indoleamine 2,3-Dioxygenase (IDO) Expression and Modulates Subsequent Inflammatory Mediators in Nasal Epithelial Cells. Acta Otolaryngol (2020) 140(2):149-56. doi: 10.1080/00016489.2019.1700304

45. Kim SB, Choi JY, Uyangaa E, Patil AM, Hossain FM, Hur J, et al. Blockage of Indoleamine 2,3-Dioxygenase Regulates Japanese Encephalitis Via Enhancement of Type I/II IFN Innate and Adaptive T-Cell Responses. J Neuroinflamm (2016) 13(1):79. doi: 10.1186/s12974-016-0551-5

46. Mazarei G, Budac DP, Lu G, Lee H, Moller T, Leavitt BR. The Absence of Indoleamine 2,3-Dioxygenase Expression Protects Against NMDA ReceptorMediated Excitotoxicity in Mouse Brain. Exp Neurol (2013) 249:144-8. doi: 10.1016/j.expneurol.2013.08.007

47. Huang YS, Ogbechi J, Clanchy FI, Williams RO, Stone TW. IDO and Kynurenine Metabolites in Peripheral and CNS Disorders. Front Immunol (2020) 11:388. doi: 10.3389/fimmu.2020.00388

48. Del Sarto JL, Rocha RPF, Bassit L, Olmo IG, Valiate B, Queiroz-Junior CM, et al. 7-Deaza-7-Fluoro-2'-C-Methyladenosine Inhibits Zika Virus Infection and Viral-Induced Neuroinflammation. Antiviral Res (2020) 180:104855. doi: 10.1016/j.antiviral.2020.104855

49. Herrera-Rios D, Mughal SS, Teuber-Hanselmann S, Pierscianek D, Sucker A, Jansen P, et al. Macrophages/Microglia Represent the Major Source of Indolamine 2,3-Dioxygenase Expression in Melanoma Metastases of the Brain. Front Immunol (2020) 11:120. doi: 10.3389/fimmu.2020.00120

50. Zhang S, Zong Y, Ren Z, Hu J, Wu X, Xiao H, et al. Regulation of Indoleamine 2, 3-Dioxygenase in Hippocampal Microglia by NLRP3 Inflammasome in Lipopolysaccharide-Induced Depressive-Like Behaviors. Eur J Neurosci (2020) 52(11):4586-601. doi: 10.1111/ejn.15016

Conflict of Interest: RD has done consultancy work for Compass Pathways, UK.

The remaining authors declare that the research was conducted in the absence of any commercial or financial relationships that could be construed as a potential conflict of interest.

Copyright (C) 2021 Marim, Teixeira, Queiroz-Junior, Valiate, Alves-Filho, Cunha, Dantzer, Teixeira, Teixeira and Costa. This is an open-access article distributed under the terms of the Creative Commons Attribution License (CC BY). The use, distribution or reproduction in other forums is permitted, provided the original author(s) and the copyright owner(s) are credited and that the original publication in this journal is cited, in accordance with accepted academic practice. No use, distribution or reproduction is permitted which does not comply with these terms. 